\title{
A negative feedback loop of ICER and NF-KB regulates TLR signaling in innate immune responses
}

\author{
Sihan $\mathrm{Lv}^{1}$, Jian $\mathrm{Li}^{1,2}$, Xinchen Qiu ${ }^{1,2}$, Weida $\mathrm{Li}^{2}$, Chao Zhang ${ }^{2}$, Zhen-Ning Zhang ${ }^{\star, 2}$ and Bing Luan ${ }^{\star, 1}$
}

The NF- $\kappa$ B pathway has important roles in innate immune responses and its regulation is critical to maintain immune homeostasis. Here, we report a newly discovered feedback mechanism for the regulation of this pathway by TLR ligands in macrophages. Lipopolysaccharide (LPS) induced the expression of ICER via p38-mediated activation of CREB in macrophages. ICER, in turn, inhibited the transcriptional activity of NF- $\kappa$ B by direct interaction with the p65 subunit of NF- $\kappa$ B. Deficiency in ICER elevated binding of NF- $\kappa$ B to promoters of pro-inflammatory genes and their subsequent gene expression. Mice deficient in ICER were hypersensitive to LPS-induced endotoxic shock and showed propagated inflammation. Whereas ICER expression in ICER KO bone marrow transplanted mice rescued the ultra-inflammation phenotype, expression of a p65 binding-deficient ICER mutant failed to do so. Our results thus establish p38-CREB-ICER as key components of a negative feedback mechanism necessary to regulate TLR-driven inflammation.

Cell Death and Differentiation (2017) 24, 492-499; doi:10.1038/cdd.2016.148; published online 23 December 2016

Activation of TLR4, a pattern recognition receptor used in the innate immune system, by lipopolysaccharide (LPS) leads to the transcription of pro-inflammatory mediators that promote innate immune responses. ${ }^{1}$ One critical pathway triggered by TLR4 is the NF- $k$ B pathway. ${ }^{2}$ TLR4 initiates a signaling cascade through adapter molecules MyD88 and TRIF, resulting in TRAF6 activation. TRAF6 in turn triggers the phosphorylation of $I_{K} \mathrm{~B}$ by $\mathrm{IKK}$ and proteosomal degradation of $\mathrm{I}_{\kappa} \mathrm{B}$. Subsequently, NF- $k$ B disassociates from $I_{k} B$ and translocates to the nucleus where it promotes the transcription of genes involved in pro-inflammatory responses. ${ }^{3,4}$ Activation of TRAF6 also leads to downstream activation of the MAPK cascade, including JNKs and p38 isoforms. ${ }^{5}$ The $\mathrm{p} 38$ pathway has attracted considerable interest as a possible target for antiinflammatory drugs. Activation of p38 results in the activation of CREB, a transcription factor that regulates diverse cellular responses including proliferation, survival and immune responses. CREB is phosphorylated and activated by MSK1/2, a downstream kinase of $\mathrm{p38}$, and subsequently mediates the transcription of genes containing a cAMP-responsive element. Several anti-inflammatory genes possess this cAMPresponsive element, including Dusp1 and IL-10. ${ }^{6}$ In addition, phosphorylated CREB has been proposed to directly inhibit NF- $\kappa \mathrm{B}$ activation by blocking the binding of CREB binding protein to the NF-kB complex, thereby limiting proinflammatory responses. ${ }^{7}$

Inducible cAMP early repressors (ICERs) are members of the cAMP-response element (CRE) modulator family and are induced by CREB. ${ }^{8}$ ICER is transcribed via an alternative internal promoter in the CREM gene and consists of four different isoforms generated by alternative splicing of its transcript (ICERI, ICERIy, ICERII, ICERII ) . ${ }^{9}$ The isoforms encode either CREB-like (ICERI) or CREM-like (ICERII) DNA-binding domains (DBDs) with or without the alternatively spliced exon $\gamma \cdot{ }^{10,11}$ ICER proteins contain DBDs but not the $\mathrm{N}$-terminal transactivation domain. This makes them function as endogenous inhibitors of gene transcription by competing with $\mathrm{CREB}$ in binding $\mathrm{CRE}$ sequences. Hence, under physiological conditions, ICER induction is a transient phenomenon that allows cAMP signaling to return to the basal state. However, whether or not ICER also mediates the anti-inflammation effect of CREB in immune responses has remained unknown.

In this study we characterized a feedback mechanism for the regulation of the NF- $\kappa \mathrm{B}$ pathway in macrophages. In this mechanism, LPS induces the expression of ICER via p38-mediated CREB activation and ICER, in turn, inhibits the transcriptional activity of NF- $\kappa$ B through direct interaction. This mechanism serves as an important feedback mechanism in preventing excessive inflammation.

\begin{abstract}
Results
LPS stimulates ICER expression via p38-CREB pathway. To test correlation of ICER expression with pathological sepsis status, mice were intraperitoneally injected with different doses $(0,10,20,30 \mathrm{mg} / \mathrm{kg})$ of LPS and mRNA levels of ICER in whole blood cells, spleen and liver were screened by quantitative real-time PCR. As shown in Figure 1a, the expression of ICER was significantly upregulated in a dose-dependent manner by LPS treatment. In mouse peritoneal macrophages (PMs), LPS also stimulated ICER mRNA levels in a time-dependent manner (Figure 1b).
\end{abstract}

\footnotetext{
${ }^{1}$ Department of Endocrinology, Shanghai Tenth People's Hospital, School of Medicine, Tongji University, Shanghai, China and ${ }^{2}$ Translational Medical Center for Stem Cell Therapy \& Institute for Regenerative Medicine, Shanghai East Hospital, School of Life Sciences and Technology, Tongji University, Shanghai, China

${ }^{*}$ Corresponding author: Bing Luan, Department of Endocrinology, Shanghai Tenth People's Hospital, School of Medicine, Tongji University, 1239 Siping Road, Shanghai 20092, China. Tel: +86 13262825126; E-mail: bluan@ @ongji.edu.cn

or Zhen-Ning Zhang, Translational Medical Center for Stem Cell Therapy \& Institute for Regenerative Medicine, Shanghai East Hospital, School of Life Sciences and Technology, Tongji University, 1239 Siping Road, Shanghai 20092, China. Tel: +86 13262825103; E-mail: znzhang @tongji.edu.cn

Received 16.9.16; revised 22.11.16; accepted 24.11.16; Edited by H Ichijo; published online 23.12.2016
} 
In addition to LPS, which activates TLR4, ligands for other TLRs as well as TNF $\alpha$ and IL- $1 \beta$ stimulated the expression of ICER in PMs (Supplementary Figure S1A and S1B).
Next, we investigated the mechanism by which ICER expression was regulated by TLR4 signaling. LPS stimulates both NF-KB and MAPK pathways in macrophages, prompting
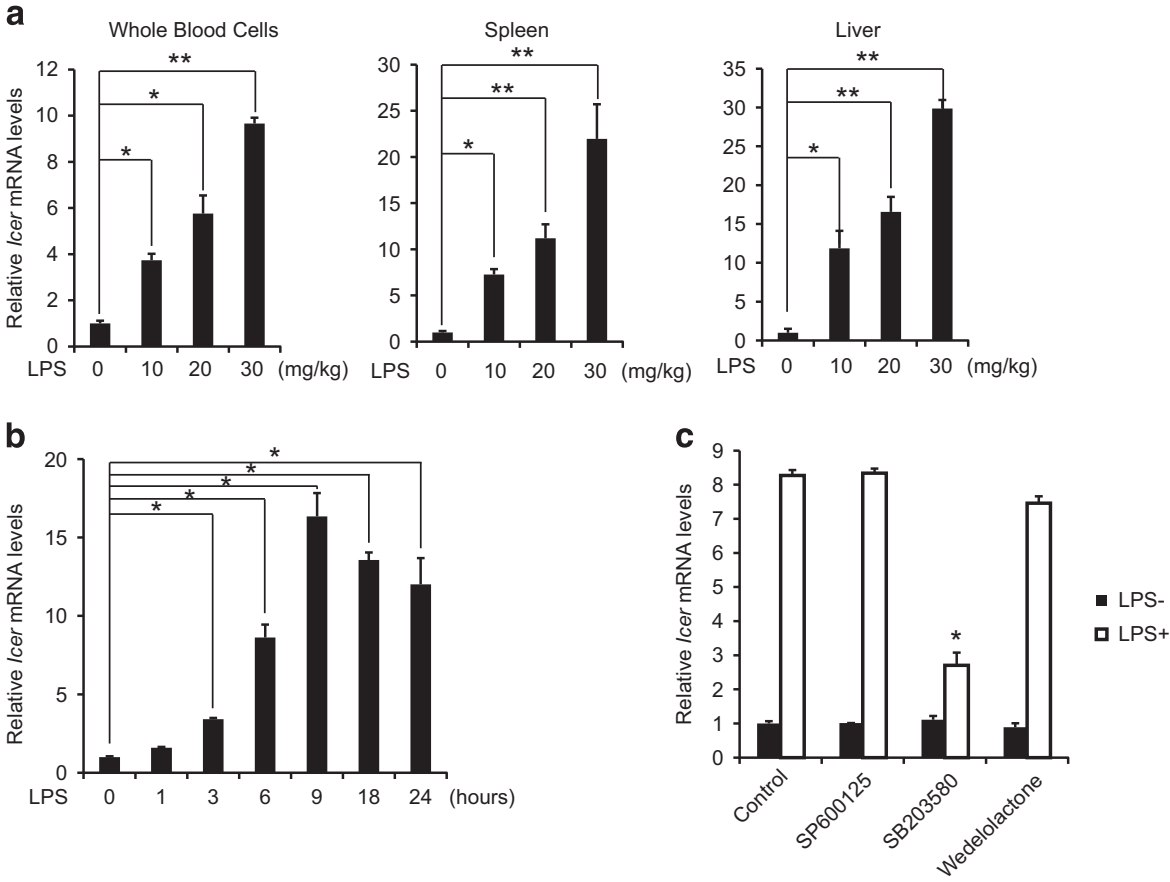

d
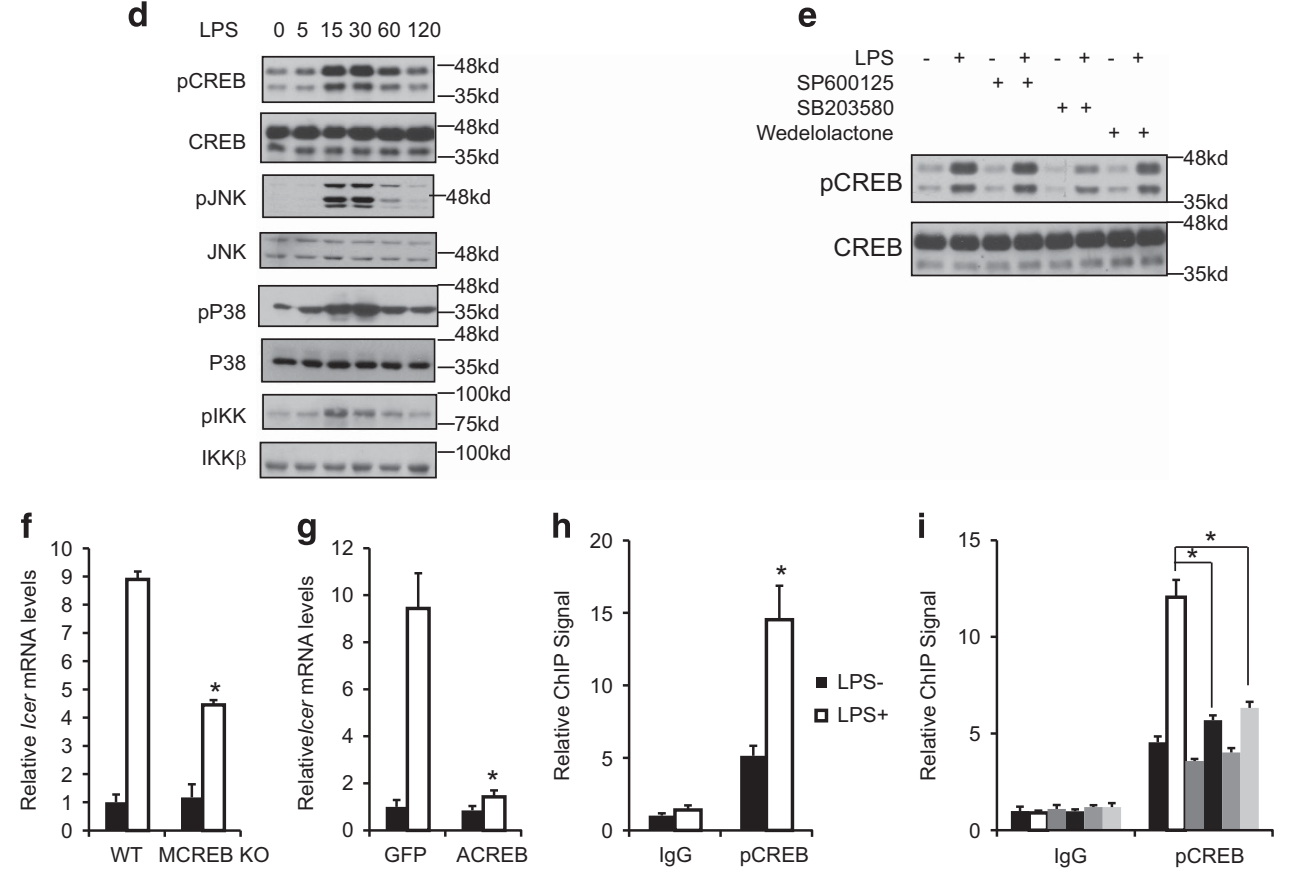

- LPS-

口LPS+

- SB203580+

- SB203580+LPS+

- SB202190+

- SB202190+LPS+

Figure 1 LPS stimulates ICER expression via p38-CREB pathway. (a) Effect of intraperitoneal (i.p.) LPS on Icer mRNA levels in peripheral whole blood cells, spleen, and liver. Data are represented as mean \pm S.E.M. ${ }^{*} P<0.05$ and ${ }^{* *} P<0.01$. (b) Effect of LPS on Icer mRNA levels in peritoneal macrophages at indicated times. Data are represented as mean \pm S.E.M. ${ }^{*} P<0.05$. (c) Effect of MAPK and IKK inhibitors on Icer mRNA levels in peritoneal macrophages. Data are represented as mean \pm S.E.M. ${ }^{\star} P<0.05$. (d) Effect of LPS on JNK, P38, IKK, and CREB phosphorylation in peritoneal macrophages. (e) Effect of MAPK and IKK inhibitors on LPS-induced CREB phosphorylation in peritoneal macrophages. (f) Effect of LPS on Icer mRNA levels in peritoneal macrophages from mice with a deletion of the CREB gene in macrophages (CREB MKO) or control littermates. Data are represented as mean \pm S.E.M. ${ }^{*} P<0.05$. (g) Effect of LPS on Icer mRNA levels in peritoneal macrophages infected with either GFP or ACREB lentivirus. Data are represented as mean \pm S.E.M. ${ }^{*} P<0.05$. (h) Chromatin immunoprecipitation (ChIP) assay of phosph-CREB recruitment over Icer promoter in peritoneal macrophages exposed to LPS. Data are represented as mean \pm S.E.M. ${ }^{*} P<0.05$. (i) Effect of $p 38$ inhibitors on phosph-CREB recruitment over Icer promoter in peritoneal macrophages exposed to LPS. Data are represented as mean \pm S.E.M. ${ }^{*} P<0.05$ 

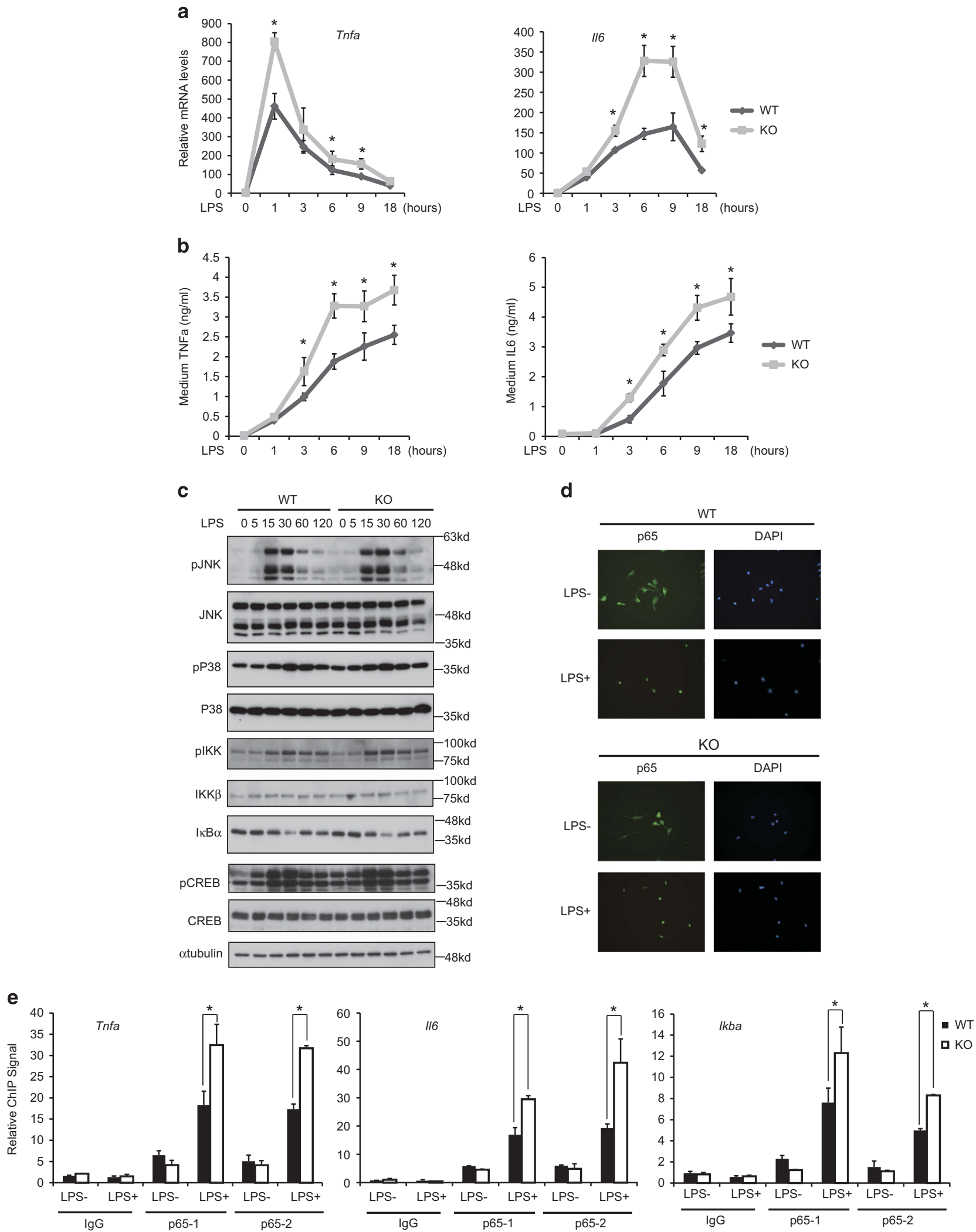

Figure 2 ICER deficiency promotes p65 DNA-binding and inflammatory responses in macrophage. (a and $\mathbf{b}$ ) Effect of LPS on pro-inflammatory genes mRNA (Tnfa and II6) levels (a) and cytokine (TNFa and IL-6) secretion in peritoneal macrophages from Icer KO mice or control littermates. Data are represented as mean \pm S.E.M. ${ }^{*} P<0.05$. (c and d) Effect of LPS on JNK, P38, IKK, and CREB phosphorylation, $I_{K} \mathrm{~B} \alpha$ degradation (c) and p65 nuclear translocation (d) in peritoneal macrophages from Icer KO mice or control littermates. (e) ChIP assay of p65 recruitment over Tnfa, 116 , and $I \kappa B \alpha$ promoters in peritoneal macrophages from Icer KO mice or control littermates with two different p65 antibodies. Data are represented as mean \pm S.E.M. ${ }^{*} P<0.05$ 
a

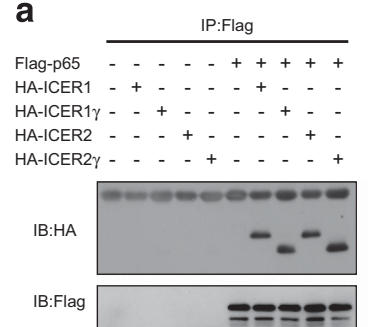

Lysate(10\%)

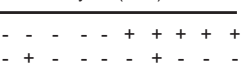

$+\ldots+\ldots+\cdots$

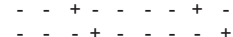

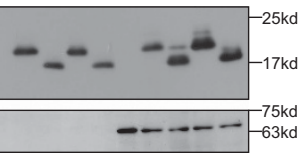

b
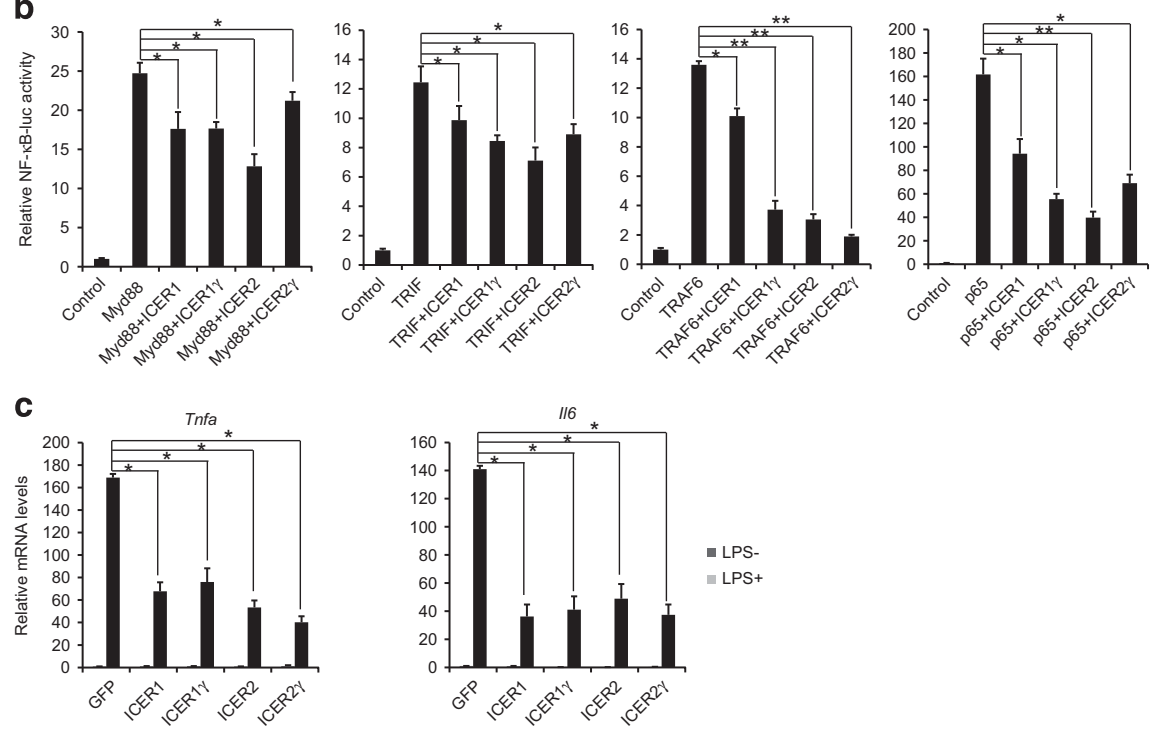

d

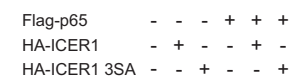

HA-ICER1 3SA - - + . +

IP:Flag $\mid \begin{aligned} & \text { IB:HA } \\ & \text { IB:Flag } \square-17 \mathrm{kd} \\ & -75 \mathrm{kd} \\ & -63 \mathrm{kd}\end{aligned}$
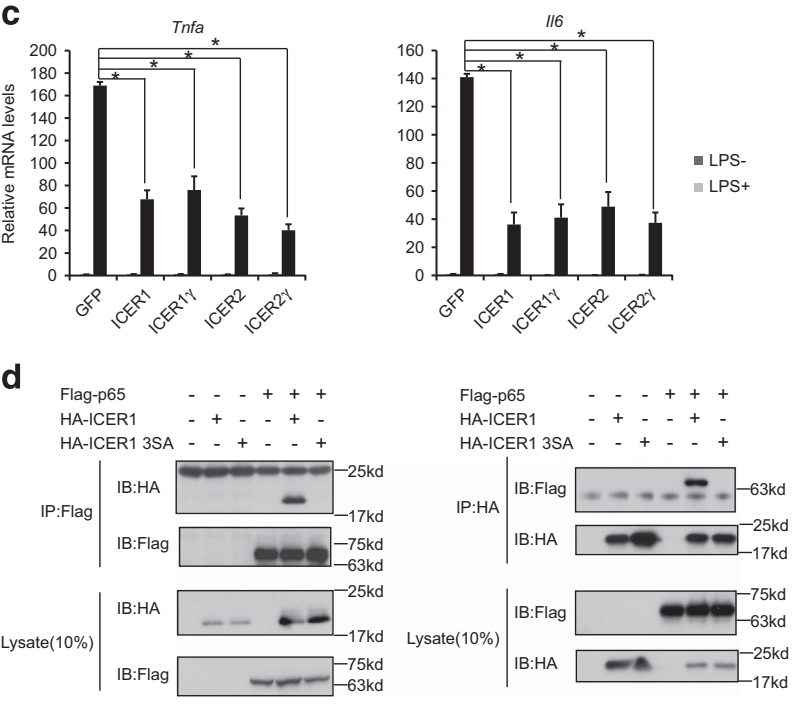

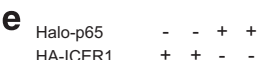
HA-ICER13SA - - + +
Anti-HA $\frac{-}{-25 \mathrm{kd}}$ Anti-HA $=--{ }_{17 \mathrm{kd}}^{-25 \mathrm{kd}}$
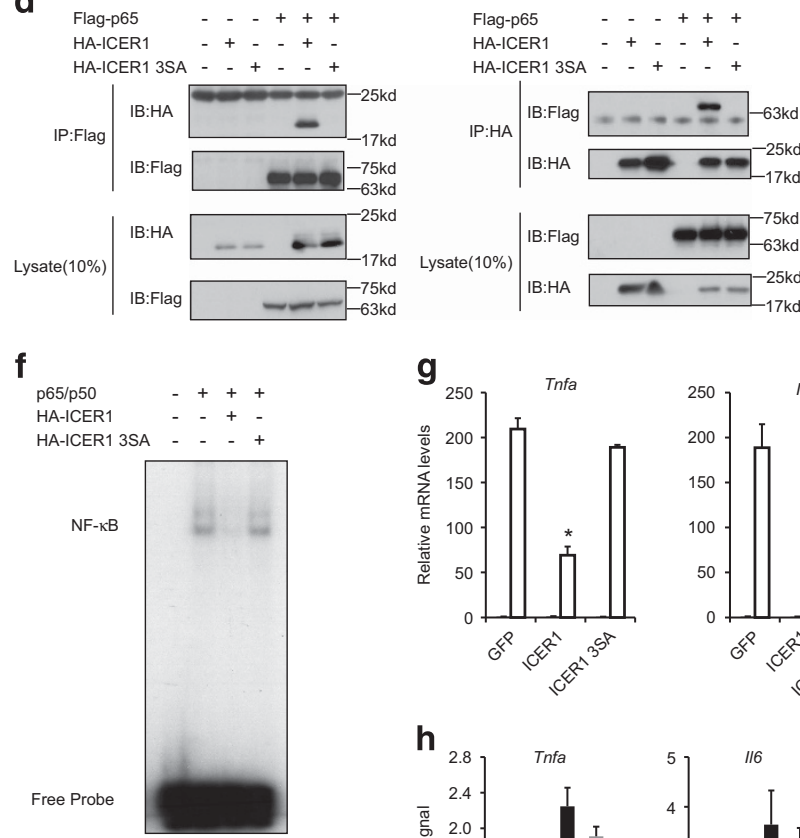

9
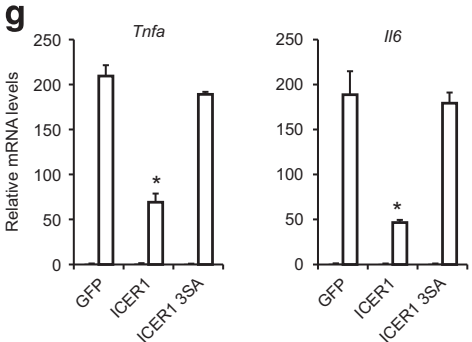

- LPS-
口 LPS+
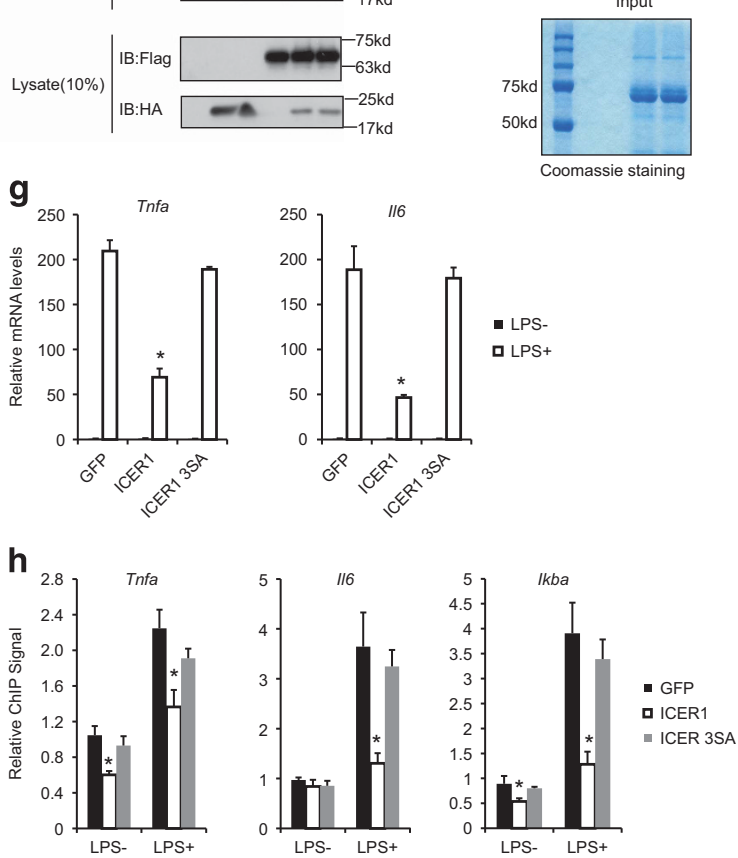

Figure 3 For caption see page 496 
us to test the effect of various NF-KB and MAPK inhibitors. Pretreatment of PMs by JNK inhibitor SP600125 or IKK inhibitor Wedelolactone had no effect on LPS-induced ICER upregulation; however, when p38 activity was inhibited by
SB203580 or SB202190, LPS-induced ICER expression was greatly impeded (Figure 1c and Supplementary Figure S2A). Meanwhile, other TLRs ligand-induced ICER upregulation was also attenuated by SB203580 or SB202190 treatment a

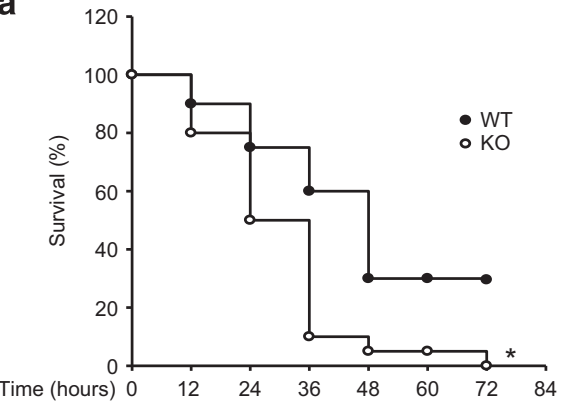

C

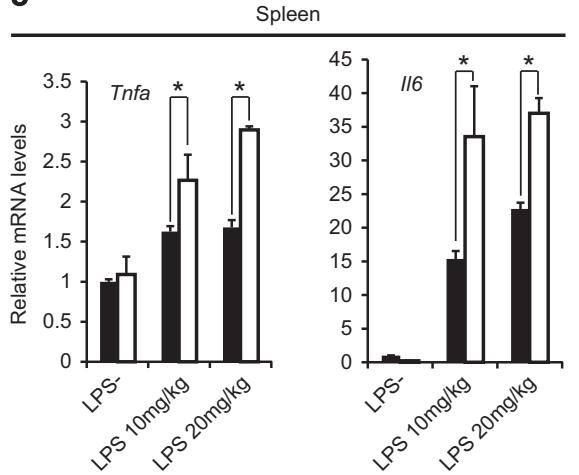

d

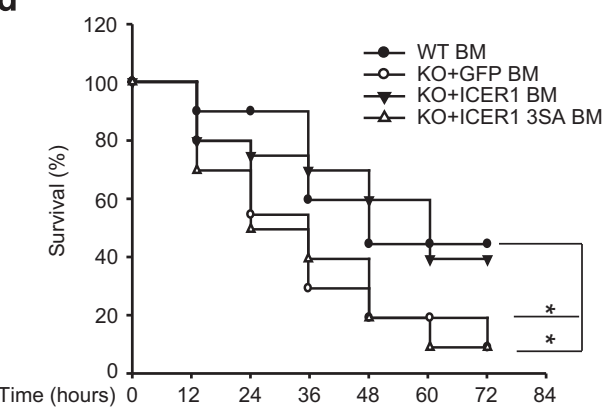

b
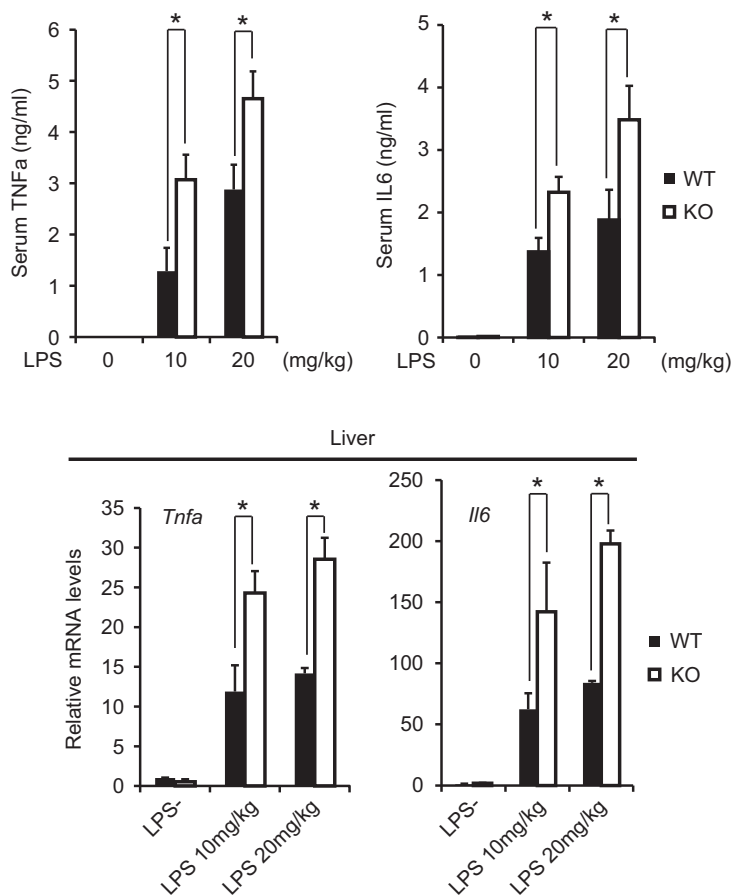

e
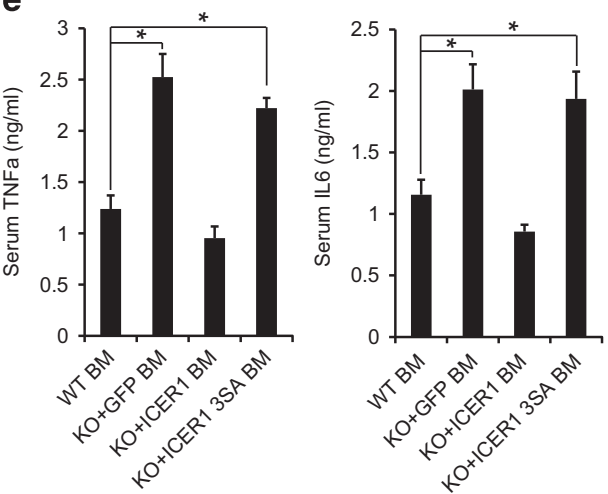

Figure 4 Anti-inflammatory effects of ICER in mice. (a) Effect of LPS i.p. (30 mg/kg) injection on survival in Icer KO mice or control littermates. Data are represented as mean \pm S.E.M. ${ }^{*} P<0.05$. (b) Effect of LPS i.p. (10 mg/kg and $20 \mathrm{mglkg}$ ) injection on circulating cytokine concentrations in $/ c e r$ KO mice or control littermates. Data are represented as mean \pm S.E.M. ${ }^{*} P<0.05$. (c) Pro-inflammatory genes (Tnfa and 116 ) mRNA levels in spleen and liver from Icer KO mice or control littermates injected with indicated amount of LPS. Data are represented as mean \pm S.E.M. ${ }^{*} P<0.05$. (d and e) Effect of LPS i.p. injection on survival $(30 \mathrm{mg} / \mathrm{kg})(\mathrm{d})$ and circulating cytokine concentrations $(20 \mathrm{mg} / \mathrm{kg})(\mathbf{e})$ in lethally irradiated mice transplanted with indicated bone marrow. Data are represented as mean \pm S.E.M. ${ }^{*} P<0.05$

Figure 3 ICER inhibits NF- $\kappa$ B activation via binding to p65. (a) Interaction between Flag-tagged $p 65$ and HA-tagged ICER isoforms in HEK293T cells. (b) Transient assay of HEK293T cells showing NF- $\kappa B$ reporter activity in cells co-transfected with ICER isoforms together with MyD88, TRIF, TRAF6, or p65. Data are represented as mean \pm S.E.M. ${ }^{*} P<0.05$ and ${ }^{* *} P<0.01$. (c) Effect of LPS on pro-inflammatory cytokine genes (Tnfa and II6) mRNA levels in peritoneal macrophages infected with either GFP or ICER isoforms lentivirus. Data are represented as mean + S.E.M. ${ }^{*} P<0.05$. (d) Interaction between Flag-tagged p65 and HA-tagged ICER1 or ICER1 3SA in HEK293T cells. (e) Pull-down assay showing interaction between purified p65 and IPed HA-tagged ICER1 or ICER1 3SA. (f) Effect of ICER1 or ICER1 3SA on p65 DNA-binding activity examined by EMSA. (g) Effect of LPS on pro-inflammatory cytokine genes (Tnfa and II6) mRNA levels in peritoneal macrophages infected with GFP, ICER1, or ICER1 3SA lentivirus. Data are represented as mean \pm S.E.M. ${ }^{*} P<0.05$. (h) ChIP assay of $p 65$ recruitment over Tnfa, Il6, and $l_{\kappa} B \alpha$ promoters in peritoneal macrophages infected with GFP, ICER1, or ICER1 $3 S A$ lentivirus. Data are represented as mean \pm S.E.M. ${ }^{*} P<0.05$. See also Supplementary Figures $S 1$ 
(Supplementary Figure S2B). P38 has been shown to promote CREB phosphorylation and activation through MSK1/2 under LPS stimulation. ${ }^{6}$ Taking into consideration the important function of CREB on ICER expression, we speculated that CREB may mediate LPS-induced ICER upregulation. Indeed, exposure of PMs to LPS increased CREB Ser133 phosphorylation and this effect was reduced by SB203580 or SB202190 (Figure 1d and e and Supplementary Figure S2C). Next, we tested the importance of CREB for LPS-induced ICER expression using homozygous CREB-floxed (CREB fl/fl) mice expressing a macrophage-specific LysM-cre transgene (MCREB KO). In PMs from MCREB KO mice, LPS-induced ICER expression was largely decreased compared with PMs from WT mice (Figure 1f). We further evaluated the role of CREB in LPS-induced ICER expression by expressing the dominant-negative CREB inhibitor ACREB, a synthetic polypeptide that selectively heterodimerizes with, and disrupts, the DNA-binding activity of all CREB family members (CREB1, CREM, and ATF1) in WT PMs. ${ }^{12}$ Similar to knocking out CREB in PMs, ACREB expression disrupted LPS-induced ICER gene expression (Figure 1g). Exposure to LPS consistently increased phospho-CREB occupancy over the ICER promoter in PMs (Figure 1h) and this effect was inhibited by SB203580 or SB202190 (Figure 1i). Taken together, these results demonstrate that the p38-CREB pathway is required for the induction of ICER expression following exposure to LPS.

ICER deficiency promotes p65 DNA-binding and inflammatory responses in macrophage. Since ICER expression was upregulated by TLRs, we evaluated the loss of function of ICER using PMs from ICER knockout mice to compare proinflammatory cytokines profiles. Notably, ICER-deficient macrophages expressed significantly higher levels of Tnfa and II-6 mRNAs when they were stimulated by LPS (Figure 2a). Consistently, deficiency in ICER resulted in more production of TNFa and IL-6 by LPS-stimulated PMs (Figure 2b). In consistent with its suppression on LPSinduced ICER gene expression, ACREB expression promoted LPS-induced Tnfa and II-6 gene expression in PMs (Supplementary Figure S3A). In contrast, exposure to Forskolin (FSK), a commonly used CREB activator through raising the levels of intracellular CAMP, further stimulated LPSinduced Icer expression in wild-type PMs but failed to do so in ICER-deficient PMs. As a result, LPS-induced Tnfa and II-6 gene expression was suppressed by FSK more dramatically in WT compared with ICER-deficient PMs (Supplementary Figure S3B). This finding suggests that ICER might be involved in the activation of negative feedback pathways downstream of p38 and CREB. To understand how ICER regulates the expression of inflammatory genes, we first examined whether or not ICER deficiency affected LPSstimulated MAPK and NF- $K B$ signaling in PMs. As shown in Figure 2c, ICER deficiency did not significantly affect the phosphorylation levels of JNK, p38, IKK, and CREB in PMs stimulated with LPS, nor did it affected the degradation of $I_{\kappa} \mathrm{B} a$ (Figure 2c). LPS-induced nuclear accumulation of the NF- $\kappa$ B subunit p65 was also not influenced by ICER deficiency (Figure 2d). These results suggest that ICER may not be involved in MAPK and NF- $K B$ signaling. Therefore, we examined the effect of ICER on the binding of p65 to the promoters of its downstream target genes by ChIP assay. Exposure to LPS increased p65 occupancy over Tnfa, II-6, and Ikba promoters more dramatically in ICER-deficient PMs compared with WT (Figure 2e), suggesting that ICER regulates the expression of NF- $\mathrm{B}$ B downstream target genes by affecting the binding of NF- $k \mathrm{~B}$ to its promoter. Taking into consideration the important function of p38 on ICER expression, we speculated that p38 may suppress the binding of NF$\kappa \mathrm{B}$ to downstream target genes promoter through ICER. Indeed, exposure of PMs to p38 inhibitor SB203580 or SB202190 promoted LPS-induced p65 occupancy over Tnfa, $I I-6$, and Ikba promoters more dramatically in WT PMs compared with ICER-deficient PMs (Supplementary Figure S3C).

ICER inhibits NF-KB activation via binding to p65. It is reported that bZIP-containing proteins such as Fos, Jun, and BZLF interact with p65 through the bZIP domain. ${ }^{13-15}$ Based on the fact that ICER also contains bZIP domain, we tested whether ICER associates with p65. Indeed, we recovered all four isoforms of HA-tagged ICER via immunoprecipitation (IP) with Flag-tagged p65 (Figure 3a). Consistent with this association, overexpression of epitope-tagged ICER decreased NF- $\kappa \mathrm{B}$ reporter activity in cells co-expressing MyD88, TRIF, TRAF6, or p65 (Figure 3b). Furthermore, when $P M s$ were infected with lentivirus expressing ICER isoforms, LPS-stimulated Tnfa and II-6 mRNAs levels were significantly decreased (Figure 3c). The PEST peptide sequence is rich in proline $(P)$, glutamic acid $(E)$, serine $(S)$, and threonine $(T)$. It has been reported that a PEST sequence in $I_{k} B$ proteins directly interacts with the p65 N-terminal domain. ${ }^{16,17}$ Based on this, we tried to identify a PEST-like sequence in the bZIP domain of ICER that may mediate its interaction with p65. We used the PESTfind analysis tool (http://emboss.bioinformatics.nl/cgi-bin/emboss/epestfind) ${ }^{18}$ to search for putative PEST sequences in ICER isoforms and found that ICER1 has a potential PEST motif (amino acids 61-72) in its bZIP domain that is conserved through all four isoforms. To further establish the interaction domain of ICER with p65, various forms of ICER1 with mutations adjacent to this potential PEST sequence: ICER1 E68/69A (2EA), ICER1 P63A (PA), ICER1 S56/59/62A (3SA) were tested for the ability to bind to p65. Flag-tagged p65 was found to associate with HA-tagged ICER1 as well as ICER1 2EA, ICER1 PA mutants; however, mutation of Ser56/59/62 to Ala (3SA) disrupted the ICER1: p65 interaction (Supplementary Figure S4A). Indeed, we were able to recover ICER1 but not ICER1 3SA via IP with Flag-tagged p65 and vice versa (Figure 3d). Next, the in vitro pull-down assay was applied to further confirm ICER-p65 interaction. Purified p65 could pull-down purified HA-tagged ICER1 but not ICER1 3SA (Figure 3e). Consistently, overexpression of either ICER1, ICER1 2EA, or ICER1 PA but not ICER1 3SA decreased NF- $\kappa$ B reporter activity in cells coexpressing p65 (Supplementary Figure S4B).

To explore whether ICER interaction with p65 directly inhibits p65 DNA-binding activity, we first mapped the region on p65 that mediated ICER binding. Deletion of N-terminus DBD of p65 resulted in complete loss of its ability to bind to ICER1. In contrast, deletion of C-terminus Transactivation domain of $\mathrm{p} 65$ had little influence on the interaction with ICER1 
(Supplementary Figure S5A and S5B). Indeed, purified ICER1 but not ICER1 3SA suppressed purified p65 DNA-binding activity as tested by EMSA (Figure 3f); strongly suggesting that ICER binds to the DBD of p65 to suppress its DNA-binding activity. As a result, lentivirus encoded expression of ICER1, but not ICER1 3SA, attenuated LPS-stimulated Tnfa and II-6 mRNAs levels in PMs (Figure 3g). Furthermore, whereas p65 occupancy over Tnfa, II-6, and Ikba promoters was dramatically inhibited by ICER1 expression, ICER1 3SA failed to show any effect (Figure 3h). Taken together, these data indicated that ICER inhibits NF- $\kappa$ B transcriptional activity via its binding to $p 65$.

Anti-inflammatory effects of ICER in mice. Intraperitoneal injection of LPS in mice can lead to endotoxic shock and mortality. Based on the results above, we predicted that the ICER KO mice would be more sensitive to LPS-induced endotoxic shock in comparison with WT mice. Indeed, when mice were given a typically sub-lethal dose of LPS, ICER KO mice were more susceptible to LPS-induced lethality than WT mice (Figure 4a). In similar experiments, we injected mice with different doses of LPS $(0,10,20 \mathrm{mg} / \mathrm{kg})$ and measured plasma cytokine concentrations after $4 \mathrm{~h}$. Notably, TNFa and IL-6 concentrations were much higher in ICER KO mice than in wild-type mice (Figure 4b). Whereas spleens and livers showed extensive inflammation after LPS injection, the elevated proinflammatory gene expression, including Tnfa and II6, was consistently more dramatic in ICER KO mice (Figure 4c). Furthermore, to avoid complications that may result from ICER expression in non-hematopoietic cells, $\gamma$-irradiated WT mice were transplanted with bone marrow (BM) from WT mice or ICER KO mice. ICER KO BM-transplanted mice were significantly more susceptible to LPS-induced septic shock in comparison with WT BM-transplanted mice as evidenced by survival curves (Figure 4d) and serum cytokine levels (Figure 4e). Notably, transplantation of ICER KO BM infected with lentivirus encoding ICER1 but not ICER1 3SA rescued this phenotype (Figure 4d and e).

Based on the effects of CREB on LPS-induced ICER expression, we evaluated the importance of CREB deficiency on LPS-induced endotoxic shock using macrophage-specific CREB knockout mice (CREB MKO). When given a sub-lethal dose of LPS, CREB MKO mice were more susceptible to LPSinduced lethality than WT mice (Supplementary Figure S6A). Serum TNF $a$ and IL-6 concentrations were much higher in ICER KO mice than in wild-type mice (Supplementary Figure S6B). Furthermore, lentivirus encoded expression of ICER1, but not ICER1 3SA, attenuated LPS-stimulated Tnfa and II-6 mRNAs levels in PMs from CREB MKO mice (Supplementary Figure S6C). These results, together with the data from ICER KO mice, suggest that CREB-ICER pathway has an important feedback role in LPS-induced NF$\kappa \mathrm{B}$ activation and acute endotoxic shock.

\section{Discussion}

CREB has critical roles in innate immune responses. CREB has been shown to inhibit NF- $\kappa$ B activity through competition for co-activators CBP/p300. ${ }^{7,19}$ CREB also has an essential role in the production of $\mathrm{IL}-10$, a potent anti-inflammatory cytokine that mediates feedback inhibition. ${ }^{6,20}$ Our previous work showed that CREB induces the M2 polarization of macrophages, an anti-inflammatory phenotype, via the induction of KLF4. ${ }^{12}$ In the present work, we describe a previously unrecognized negative feedback mechanism for the regulation of NF- $\kappa$ B by TLR ligands via CREB-induced ICER. In this mechanism, ICER expression is upregulated by TLR ligands via p38-mediated CREB activation, and ICER, in turn, inhibits NF- $k$ B transcriptional activity by interaction with the NF- $k B$ p65 subunit. This mechanism has an important role in limiting inflammation both in vitro and in vivo. Thus, a systematic investigation into the role of CREB in innate immune function would likely lead to new therapeutic strategies for manipulation of inflammatory responses.

Owing to the use of the alternative P2 promoter located in the middle of the CREM gene, ICER lacks the upstream transactivation domain of CREM and thus fails to recruit co-activators such as CBP/p300. ${ }^{8}$ ICER is better known as a repressor for CREB, c-Fos, or c-Jun-mediated transcription. ${ }^{8,21,22}$ ICER could also bind to NF-AT ref. 23 and AP1 ref. 24 inhibiting their transcriptional activity. Here, we report NF- $\kappa \mathrm{B}$ as a new class of transcriptional factors that are negatively regulated by ICER, further expanding our fundamental understanding of this repressor. It is tempting to speculate that ICER may serve as a universal transcriptional suppressor that has a broad impact on multiple signal pathways and organ functions under specific physiological conditions. This prompts further study by both our laboratory and others.

\section{Materials and Methods}

Animals. In total, 8-10-week-old and weight-matched male C57BL/6 J mice were purchased from Shanghai Laboratory Animal Center (Shanghai, China) and were adapted to colony cages with $12 \mathrm{~h}$ light/dark cycle in a temperature-controlled environment with free access to water and standard irradiated rodent diet (5\% fat; Research Diet, D12450, New Brunswick, NJ, USA). ICER knockout mice were kindly provided by Dr. Shogo Endo (Tokyo Metropolitan Geriatric Hospital and Institute of Gerontology). Macrophage-specific knockout of CREB was provided by Dr. Marc Montminy (Salk Institute, La jolla, CA, USA) as described. ${ }^{12}$

Cells, antibodies, and reagents. Mouse PMs were prepared in our laboratory following procedure described previously. ${ }^{25}$ In brief, C57BL/ $6 \mathrm{~J}$ or ICER knockout mice and littermates were injected with $3 \%$ sterile thioglycollate (Sigma, St. Louis, MO, USA) intraperitoneally ( $3 \mathrm{ml}$ per mouse). After 3 days, mice were euthanized, and RPMI medium was injected intraperitoneally and then retrieved. The cells were then treated with red blood cell lysis buffer $\left(0.15 \mathrm{M} \mathrm{NH}_{4} \mathrm{Cl}, 10.0 \mathrm{mM}\right.$ $\mathrm{KHCO}_{3}$, and $0.1 \mathrm{mM} E D T A$ ) and washed with RPMI medium. The purity of the macrophages was confirmed by flow cytometry. HEK293T cells were obtained from the American Type Culture Collection (ATCC) and maintained in DMEM with $10 \%$ fetal bovine serum. Anti-pJNK1/2 (Thr183/Tyr185), JNK1/2, pP38 (Thr180/Tyr182), $\mathrm{P} 38$, plKK $\alpha / \beta$ (Ser176/Ser180), І $\kappa \mathrm{B} \alpha$, p65 antibodies were purchased from Cell signaling Technology (Danvers, MA, USA). Anti- $\alpha$-tubulin, p65 antibody was purchased from Abcam (Cambridge, UK). Anti-pCREB (Ser133) and CREB antibodies were purchased from Abmart (Berkeley Heights, NJ, USA). LPS was purchased from Sigma. Pam3Csk4, poly (I:C), and CpG-ODN were from InvivoGen (San Diego, CA, USA). SP600125, SB203280 were from medchemexpress. Wedelolactone was from Sanra Cruz Biotech (Dallas, TX, USA).

Cytokine analysis. Mice were injected with LPS for $4 \mathrm{~h}$ and serum was obtained through cardiac puncture. Serum levels of TNF $\alpha$ and IL- 6 were determined using Mouse TNF $\alpha$ and IL-6 ELISA kit from ebioscience (San Diego, CA, USA).

ChIP and quantitative real-time-PCR. Cells were plated in 150-mm plates and exposed to LPS (10ng/ml) for $1 \mathrm{~h}$. ChIP assays were performed as described. ${ }^{26}$ Total RNA was isolated by using TRIzol reagent (Invitrogen, Carlsbad, CA, USA) and 
reverse transcription was done using FastQuant RT kit (Tiangen, Shanghai, China). Real-time PCR was carried out using SuperReal SYBR Green kit (Tiangen) and Lightcycler 96 (Roche, Penzberg, Germany). $\beta$-actin was used as a reference gene. Primer sequences used for real-time PCR:

Tnf $\alpha$ forward 5'-TGAACTTCGGGGTGATCGGTC-3'

Tnfo reverse $5^{\prime}$-AGCCTTGTCCCTTGAAGAGGAC-3

116 forward 5'-GACTTCACAGAGGATACCAC-3'

$1 / 6$ reverse $5^{\prime}$-TCTCTCTGAAGGACTCTGGC-3'

Icer forward 5'-TGGACTGTGGTACGGCCAAT-3

Icer reverse $5^{\prime}$-CAGTTTCATCTCCAGTTACA-3'

$\beta$-actin forward 5'-GTGACGTTGACATCCGTAAAGA-3'

$\beta$-actin reverse $5^{\prime}$-GCCGGACTCATCGTACTCC-3'

Primer sequences used for ChIP:

Icer promoter Forward 5'-AGGGCTTTGCTTTCAGTGAG-3'

Icer promoter reverse 5'-CTTGTTGGCCGTACCACAGT-3'

Tnfo promoter forward $5^{\prime}$-CCCCAGATTGCCACAGAATC- $3^{\prime}$

Tnfo promoter reverse $5^{\prime}$-CCAGTGAGTGAAAGGGACAG-3'

116 promoter forward $5^{\prime}$-AGCTACAGACATCCCCAGTCTC-3'

116 promoter reverse $5^{\prime}$-TGTGTGTCGTCTGTCATGCG-3'

I $k$ ba promoter forward 5'-AAATCTCCAGATGCTACCCGAGAG-3

I $\kappa$ ba promoter reverse 5'-ATAATGTCAGACGCTGGCCTCCAA-3'

Blotting and immunostaining. Immunoblot, IP, and immunostaining assays were performed as described. ${ }^{26}$ The image was caught by Tanon-5500 Chemiluminescent Imaging System (Tanon, China).

Luciferase reporter assay. HEK293T cells were transfected with NF- $\kappa \mathrm{B}$-Luc reporter, RSV- $\beta$ gal, and indicated plasmids for $48 \mathrm{~h}$ and luciferase assays were performed. $^{26}$

BM transplantation. BM cells were flushed from the tibias and femurs of mice using RPMI supplemented with 10\% FBS and penicillin-streptomycin. After RBC lysis, the cells were washed and resuspended in $1 \mathrm{ml}$ PBS containing $10^{7}$ cells, after infection with $10^{8}$ lentivirus, $200 \mu$ l of BM cells was injected intravenously into lethally irradiated (10 Gy) recipients.

Statistical analysis. All studies were performed on at least three independent occasions. Results are reported as mean \pm s.e.m. The comparison of different groups was carried out using two-tailed unpaired Student's t-test or two-way ANOVA test. Differences were considered statistically significant at ${ }^{*} P<0.05$ and ${ }^{*} P<0.01$.

Ethics statement. All animal studies were approved by the Animal Experiment Committee of Tongji University and in accordance with the guidelines of School of medicine, Tongji University (Permit Number: TJ0027 Rev2). This study was carried out in strict accordance with the recommendations in the Guide for the Care and Use of Laboratory Animals of the Ministry of Science and Technology of the People's Republic of China.

\section{Conflict of Interest}

The authors declare no conflict of interest.

Acknowledgements. We thank Dr. Marc Montminy and Susan Hedrick (Salk Institute) for discussion and technical support. We thank Dr. Shogo Endo (Tokyo Metropolitan Geriatric Hospital and Institute of Gerontology) for providing us with ICER KO mice. We thank Dr. Jerome V Karpiak (University of California San Diego School of Medicine, Sanford Consortium for Regenerative Medicine, La Jolla, CA 92037, USA) for English-language editing.

\section{Author contributions}

$\mathrm{SL}, \mathrm{JL}, \mathrm{XQ}, \mathrm{ZNZ}$, and $\mathrm{BL}$ designed and conducted the experiment and analyzed the data. BL wrote the manuscript. WL, CZ, and ZNZ designed the experiments and edited the manuscript. All authors read and approved the manuscript. BL is responsible for the integrity of the work as a whole.

1. Akira S, Hemmi H. Recognition of pathogen-associated molecular patterns by TLR family. Immunol Lett 2003; 85: 85-95.

2. Miggin SM, O'Neill LA. New insights into the regulation of TLR signaling. J Leukoc Biol 2006 80: 220-226.

3. Moresco EM, LaVine D, Beutler B. Toll-like receptors. Curr Biol 2011; 21: R488-R493.

4. Kawai T, Akira S. TLR signaling. Semin Immunol 2007; 19: 24-32.

5. Han J, Lee JD, Bibbs L, Ulevitch RJ A. MAP kinase targeted by endotoxin and hyperosmolarity in mammalian cells. Science 1994; 265: 808-811.

6. Ananieva O, Darragh J, Johansen C, Carr JM, Mcllrath J, Park JM et al. The kinases MSK1 and MSK2 act as negative regulators of Toll-like receptor signaling. Nat Immunol 2008; 9: 1028-1036.

7. Parry GC, Mackman N. Role of cyclic AMP response element-binding protein in cyclic AMP inhibition of NF-kappaB-mediated transcription. J Immunol 1997; 159: 5450-5456.

8. Molina CA, Foulkes NS, Lalli E, Sassone-Corsi P. Inducibility and negative autorequlation of CREM: an alternative promoter directs the expression of ICER, an early response repressor. Cell 1993; 75: 875-886

9. Foulkes NS, Borrelli E, Sassone-Corsi P. CREM gene: use of alternative DNA-binding domains generates multiple antagonists of cAMP-induced transcription. Cell 1991; 64: 739-749.

10. Stehle JH, Foulkes NS, Molina CA, Simonneaux V, Pevet P, Sassone-Corsi P. Adrenergic signals direct rhythmic expression of transcriptional repressor CREM in the pineal gland. Nature 1993; 365: 314-320.

11. Laoide BM, Foulkes NS, Schlotter F, Sassone-Corsi P. The functional versatility of CREM is determined by its modular structure. EMBO $\mathrm{J}$ 1993; 12: 1179-1191.

12. Luan B, Yoon YS, Le Lay J, Kaestner KH, Hedrick S, Montminy M. CREB pathway links PGE2 signaling with macrophage polarization. Proc Natl Acad Sci USA 2015; 112 15642-15647.

13. Gutsch DE, Holley-Guthrie EA, Zhang Q, Stein B, Blanar MA, Baldwin AS et al. The bZIP transactivator of Epstein-Barr virus, BZLF1, functionally and physically interacts with the p65 subunit of NF-kappa B. Mol Cell Biol 1994; 14: 1939-1948.

14. Stein B, Baldwin AS Jr., Ballard DW, Greene WC, Angel P, Herrlich P. Cross-coupling of the NF-kappa B p65 and Fos/Jun transcription factors produces potentiated biological function. EMBO J 1993; 12: 3879-3891.

15. Hu CD, Chinenov Y, Kerppola TK. Visualization of interactions among bZIP and Rel family proteins in living cells using bimolecular fluorescence complementation. Mol Cell 2002; 9 789-798.

16. Sue SC, Dyson HJ. Interaction of the IkappaBalpha C-terminal PEST sequence with NFkappaB: insights into the inhibition of NF-kappaB DNA binding by IkappaBalpha. J Mol Bio 2009; 388: 824-838.

17. Ernst MK, Dunn LL, Rice NR. The PEST-like sequence of I kappa $B$ alpha is responsible for inhibition of DNA binding but not for cytoplasmic retention of c-Rel or RelA homodimers. Mol Cell Biol 1995; 15: 872-882.

18. Chepelev NL, Bennitz JD, Huang T, McBride S, Willmore WG. The Nrf1 CNC-bZIP protein is regulated by the proteasome and activated by hypoxia. PloS One 2011; 6: e29167.

19. Ollivier V, Parry GC, Cobb RR, de Prost D, Mackman N. Elevated cyclic AMP inhibits NFkappaB-mediated transcription in human monocytic cells and endothelial cells. J Biol Chem 1996: 271: 20828-20835.

20. Saraiva M, O'Garra A. The regulation of IL-10 production by immune cells. Nat Rev Immunol 2010; 10: 170-181.

21. Ahn S, Olive M, Aggarwal S, Krylov D, Ginty DD, Vinson C. A dominant-negative inhibitor of CREB reveals that it is a general mediator of stimulus-dependent transcription of c-fos. Mol Cell Biol 1998; 18: 967-977.

22. Masquilier D, Sassone-Corsi P. Transcriptional cross-talk: nuclear factors CREM and CREB bind to AP-1 sites and inhibit activation by Jun. J Biol Chem 1992; 267 22460-22466.

23. Bodor J, Habener JF. Role of transcriptional repressor ICER in cyclic AMP-mediated attenuation of cytokine gene expression in human thymocytes. J Biol Chem 1998; 273: 9544-9551.

24. Mioduszewska B, Jaworski J, Kaczmarek L. Inducible CAMP early repressor (ICER) in the nervous system-a transcriptional regulator of neuronal plasticity and programmed cell death. $J$ Neurochem 2003; 87: 1313-1320.

25. Sun J, Luan Y, Xiang D, Tan X, Chen H, Deng $Q$ et al. The $11 \mathrm{~S}$ proteasome subunit PSME3 is a positive feedforward regulator of NF-kappaB and important for host defense against bacterial pathogens. Cell Rep 2016; 14: 737-749.

26. Luan B, Goodarzi MO, Phillips NG, Guo X, Chen YD, Yao J et al. Leptin-mediated increases in catecholamine signaling reduce adipose tissue inflammation via activation of macrophage HDAC4. Cell Metab 2014; 19: 1058-1065. 